\title{
The Interplay between Alcoholic Liver Disease, Obesity, and the Metabolic Syndrome
}

\author{
Stephen Malnick ${ }^{a}$ Yaakov Maor $^{\mathrm{b}}$ \\ ${ }^{a}$ Department of Internal Medicine C, Kaplan Medical Center, Rehovot, Israel; ${ }^{b}$ Institute of Gastroenterology and \\ Hepatology, Kaplan Medical Center, Rehovot, Israel
}

\section{Keywords}

Alcohol · Fatty liver $\cdot$ Metabolic syndrome

\begin{abstract}
Background: Fatty liver may be the result of several factors. The two main contributors are nonalcoholic fatty liver disease (NAFLD) and alcoholic liver disease (ALD). Summary: NAFLD is the hepatic manifestation of the metabolic syndrome (MetS) and is the major cause of chronic liver disease worldwide as a result of the obesity epidemic. ALD is also a common cause of chronic liver disease. Obesity is a major contributory factor to MetS and is also common in individuals who consume large amounts of alcohol. There is a similar hepatic pathology and both can result in severe fibrosis, cirrhosis, and its complications including hepatocellular carcinoma. This review discusses the etiology, pathogenesis, and genetics of both NAFLD and ALD and their interaction. It is necessary to understand this better in order to prevent and treat these important causes of liver disease worldwide. Key Message: Obesity, MetS, and alcohol consumption are linked to the development and progression of fatty liver disease. The coexistence of these factors in many patients requires a reassessment of many aspects of treatment of fatty liver disease.

(c) 2020 S. Karger AG, Basel
\end{abstract}

Affiliated to the Hebrew University, Jerusalem, Israel.

\section{Introduction}

Hepatic steatosis is defined as intrahepatic triacylglycerol of at least $5 \%$ of liver weight [1]. The most common conditions responsible for hepatic steatosis are nonalcoholic fatty liver disease (NAFLD) and alcoholic liver disease (ALD). NAFLD is the hepatic manifestation of the metabolic syndrome (MetS) and, as a consequence of the epidemic of obesity, is now the commonest cause of cirrhosis and its complications including hepatocellular carcinoma (HCC) [2]. ALD occurs as a result of daily consumption of alcohol of $20 \mathrm{~g}$ daily for women and of $30 \mathrm{~g}$ daily for men [3].

The global burden of ALD has been difficult to assess due to wide country to country variations and trends in the reported prevalence. The worldwide average consumption is $6.2 \mathrm{~L}$ of $100 \%$ ethanol per adult per year [4]. The highest consumption is in the Eastern European countries around Russia (11.9 L per adult), other areas of Europe (11.9 L per adult), and the Americas with $8.7 \mathrm{~L}$ per adult. In 2012, 139 million disability-associated life years (DALY) and 5.9\% of all global deaths were attributable to alcohol consumption [5].

The global prevalence of alcohol use disorders (AUD) is $4.1 \%$ and alcohol use-dependence is 3.0\% [6]. Cirrhosis is caused by many factors including excess alcohol consumption. One of the complications of cirrhosis is liver cancer. Cirrhosis has been estimated to cause 1.16 million 
deaths worldwide and 788,000 deaths from liver cancer. Together they account for $3.5 \%$ of all deaths worldwide [6].

NAFLD is the hepatic manifestation of MetS [2]. The prevalence of obesity has increased 6-fold over the last four decades [7], resulting in a rapid growth in the prevalence of NAFLD which is now the major cause of cirrhosis worldwide [6]. Approximately $25 \%$ of the world population has NAFLD $[8,9]$. Although NAFLD is linked to obesity and diabetes, there is not always a correlation between NAFLD prevalence and areas of increased caloric consumption and availability, implying that other factors may be involved in the development and progression of NAFLD [10].

NAFLD is a multi-factorial disease resulting from a complex of environmental "hits" and genetic backgrounds. Both the development and progression of NAFLD are strongly associated with insulin resistance and MetS, especially abdominal obesity and type 2 diabetes mellitus [11]. NAFLD encompasses a spectrum of pathology ranging from steatosis, which has a benign prognosis, through steatohepatosis to fibrosis and cirrhosis and may result in HCC [12]. It has been estimated that the mean fibrosis progression rate per year for patients with biopsy-proven NASH is 0.09 and that $40 \%$ of these patients will progress to more advanced degrees of fibrosis [13]. The annual incidence of HCC in patients with NAFLD is 0.445 per 1,000 person-years and 5.3 per 1,000 person-years in patients with NASH. In addition, patients with NAFLD have a two times higher risk of liver-related mortality and an increased risk for cardiovascular events both fatal and nonfatal [13].

Both ALD and NAFLD have a significant effect on the global burden of disease. They may be present in the same individual and result in more advanced hepatic pathology and a worse outcome. Furthermore, alcohol consumption may accelerate the development of MetS, and NAFLD results in hepatic damage by increasing endogenous alcohol production [14]. It is the purpose of this review to discuss the interaction between ALD and NAFLD.

\section{Similarities and Dissimilarities between ALD and NAFLD}

ALD and NAFLD are the two most common types of chronic liver disease in Western populations $[3,15]$. Both conditions gradually progress from simple steatosis to steatohepatitis, fibrosis, and cirrhosis. However, only 15$20 \%$ of heavy alcohol drinkers ever develop liver cirrhosis, and liver fibrosis occurs and progresses in NAFLD in only a minority and at a highly variable rate $[3,15]$. Based on the present data, international liver societies arbitrarily established a gender-specific threshold of average alco- hol intake of $30 \mathrm{~g} / \mathrm{day}$ for men and $20 \mathrm{~g} /$ day for women [15]. Equivalent thresholds for BMI or waist circumference, the most common predisposing factors for NAFLD, have not been proposed; furthermore, a significant proportion of patients with NAFLD are lean [8]. Although the metabolic factors associated with NAFLD are well known, only a few studies have examined which metabolic factors are most strongly associated with advanced liver disease. In this regard, variables, such as age, male gender, AST/ALT ratio, and presence of diabetes or impaired fasting glucose predict more accurately progression to cirrhosis in patients with NAFLD [10]. Likewise, age, BMI, female sex, blood glucose level, and hepatic iron accumulation have emerged as independent risk factors for fibrosis in alcohol-induced liver disease [16].

ALD differs from NAFLD in one unique clinical condition, namely, acute alcoholic hepatitis. This most severe form of ALD is associated with a very high short-term mortality and does not occur in patients with NAFLD, unless they also consume alcohol excessively [17].

An additional difference between NAFLD/NASH and alcohol-associated HCC is that patients with NAFLD/ NASH may develop HCC without apparent cirrhosis [18]. A report of 162 individuals with NAFLD/NASHassociated HCC found that almost $42 \%$ had no evidence of cirrhosis [18]. In contrast alcohol-associated HCC was highly associated with cirrhosis. Ertl et al. [18] suggest that NAFLD/NASH as the hepatic manifestation of MetS may itself pose a risk factor for HCC, even in the absence of cirrhosis. A summary of the comparison between NAFLD and ALD is shown in Table 1.

The resemblance of the histological features of ALD and NAFLD makes it harder to distinguish between ALD and NAFLD [19]. However, several hallmarks may be suggestive of an alcoholic etiology rather than nonalcoholic (i.e., high density of Mallory-Denk bodies, central hyaline sclerosis, lipogranulomas, and cholestasis). Moreover, differences in inflammatory cell infiltration, characterized by a higher rate of neutrophils surrounding ballooned hepatocytes and portal inflammation and necrosis, are more common in ALD than NAFLD [20].

Histopathological similarities clearly suggest possible common pathophysiologic pathways for both conditions. In ALD and NAFLD, animal and human studies observed mitochondrial dysfunction and upregulation of the microsomal CYP2E1 as key elements in the progression from steatosis to steatohepatitis. A growing number of genetic variants have been associated with the pathogenesis of NAFLD and ALD. Furthermore, these variants are associated with disease progression to advanced liver disease and with development of HCC [21]. The same genetic variants play a role both in NAFLD and in ALD. Changes of microbiome composition in the gut of patients with NAFLD and ALD have been consistently de- 
Table 1. Comparison between NAFLD and ALD

\begin{tabular}{|c|c|c|}
\hline & NAFLD & ALD \\
\hline Alcohol intake, g/day & $\leq 30$ for men, 20 for women & No safe limit including binge drinking \\
\hline Effect of alcohol & Increases hepatic injury & Contributes to the development of MetS \\
\hline Clinicopathological picture & $\begin{array}{l}\text { Steatosis progressing through steatohepatitis } \\
\text { to fibrosis and cirrhosis }\end{array}$ & $\begin{array}{l}\text { Acute alcoholic hepatitis, ALD - steatosis progressing } \\
\text { to cirrhosis } \\
\text { Cholestatic hepatitis }\end{array}$ \\
\hline Development of HCC & Often independent of cirrhosis & Usually after development of cirrhosis \\
\hline MetS & Strong risk factor & Poor prognostic factor \\
\hline Cardiovascular disease & Higher incidence & Females $>60$ years some protective effect \\
\hline Global burden of disease & Significant & Significant \\
\hline Genetic variants & PNPLA3 TM6SF2 & PNPLA3 TM6SF2 \\
\hline Disease interaction & $\begin{array}{l}\text { Obesity is a risk factor for progression } \\
\text { of ALD }\end{array}$ & $\begin{array}{l}\text { Alcohol consumption increases weight and increases } \\
\text { the prevalence of MetS } \\
\text { Maybe J type curve for moderate alcohol } \\
\text { consumption and liver disease }\end{array}$ \\
\hline
\end{tabular}

tected. This microbial population can then produce an array of signaling molecules that regulate metabolic and inflammatory pathways [22]. These topics will be further discussed.

\section{The Gut Microbiome in Alcoholic and Nonalcoholic Liver Disease}

The human gut microbiota consists of bacteria, viruses, and fungi [23]. The recent application of next-generation DNA sequencing has enabled culture-independent identification of the components of the fecal microbiome [24]. These techniques include sequencing of the ribosomal 16S RNA and metagenomic shotgun techniques.

The maternal microbiota is responsible for the first colonization of the newborn intestine, and following birth, the diversity increases with the adult composition being acquired by the age of 3-5 years [25]. There are changes in the microbiome in the elderly [26]. The majority of the variation of the microbiome is related to environmental factors, including diet, antibiotic treatment, physical activity, and bowel motion frequency rather than genetics.

The microbiota has been examined in patients with ALD. This has produced differing results, which have been summarized by Bajaj [23]. In addition, only about $15-20 \%$ of those with AUD develop ALD [27]. There is evidence to suggest that other factors, such as psychiatric factors, including depression and changes in the circadian rhythm, may be confounding factors [28]. A comparison of the stool microbiome of 18 healthy individuals to that of 48 patients with AUD (19 of whom with ALD) found that the abundance of serum levels of endotoxin were higher in all patients with AUD, but there was no difference between those with and without ALD. In addition, the microbiome of colonic biopsy samples was examined by multitag pyrosequencing. There was a subgroup that had a dysbiosis with a lower abundance of Bacteroidetes and a higher abundance of Proteobacteria [29]. Overall, there was a poor correlation between colonic mucosal dysbiosis and ALD. In addition, there have been changes in the metabolome of the fecal mucosa in AUD patients compared to healthy controls. A study of $60 \mathrm{pa}-$ tients with AUD found that only $40 \%$ of the patients had a dysbiosis with a decrease in the abundance of Ruminococcaceae and an increased intestinal permeability. After 3 weeks of abstinence from alcohol, there was an increase in the abundance of Ruminococcaceae. Furthermore, the levels of bacteria, as assessed by quantitative PCR, which were lower with AUD, returned to the baseline level. There was also a correlation between increased intestinal permeability and higher levels of depression, anxiety, and craving for alcohol even after withdrawal [30]. It remains to be observed if this could predict those individuals with an increased likelihood of relapse.

In addition, changes in the fecal metabolome of AUD patients compared to healthy controls, including different levels of short-chain fatty acids and a decrease in antioxidant fatty acids, have been observed [31].

There is an interaction between the intestinal mucosal surface and the microbiome. The gut has a barrier function, protecting the host from the microbiome by reducing the contact between the microbiome and the epithe- 
lial cells and confining the bacteria to the intestine [32] Alcohol has been shown to alter the function of the gut barrier, resulting in increased intestinal permeability and translocation of luminal antigens (reviewed in [33]).

The microbiome has also been found to have an important role in NAFLD. The microbiome of patients with NAFLD is different from healthy controls with a lower abundance of Firmicutes and Faecalibacterium and a higher abundance of Parabacteroides and Allisonella in their fecal microbiome [34]. A decrease in intrahepatic fat content has been linked to a lower abundance of Firmicutes and a higher abundance of Bacteroidetes [34]. Fecal transplantation from mice with NAFLD to control mice resulted in NAFLD in those mice receiving the transplantation [35]. The severity of NAFLD has also been shown to be associated with both a gut dysbiosis and a change in the metabolic function of the gut's microbiota [36]. It has even been suggested that the microbiomal profile could serve as a surrogate for determining the degree of hepatic fibrosis [37].

Small bowel bacterial overgrowth (SIBO) together with gut barrier dysfunction are present in NAFLD [38]. In mice fed a high-fat diet, there is a decrease in tight junction proteins in the proximal small intestine, resulting in increased intestinal permeability and elevation of the level of serum endotoxins in the portal vein [39]. This is also present in humans as shown by a report of 53 NAFLD patients compared to 32 healthy subjects showing both widened tight junctions and decreased immunostaining for occludin in the proximal small bowel mucosa, and in addition, increased levels of TNF- $\alpha$, IL-6, and interferon- $\gamma$ [40].

\section{Genetics in NAFLD and ALD}

Evidence for a shared pathophysiology and the central role of lipid metabolism in both ALD and NAFLD may be derived from genetic studies. A genome-wide (GWAS) approach has identified genetic risk loci for ALD and NAFLD and has demonstrated that the severity and progression of both NAFLD and ALD are influenced by a number of the same genetic variants [21,41]. These include a common nonsynonymous variant (rs738409 c.444 $\mathrm{C}>\mathrm{G}$ p.I148M) in the patatin-like phospholipase domaincontaining 3 (PNPLA3) gene that encodes adiponutrin, a protein involved in lipid metabolism, which has been shown to influence the severity of steatosis, steatohepatitis, fibrosis, and HCC risk in NAFLD and ALD [42, 43].

Subsequently, a variant in the transmembrane 6 superfamily member 2 (TM6SF2) gene (rs5854296 c.449 C>T, p.E167K) was associated with increased severity of liver disease in NAFLD and ALD [41, 42]. More recently, the rs641738 variant in the membrane-bound O-acyltransferase domain-containing 7/transmembrane channel- like 4 (MBOAT7/TMC4) locus has been related to a higher risk of cirrhosis in alcohol abusers and with liver disease progression in NAFLD [41, 42].

There are, however, several notable differences in the prevalence of gene polymorphisms between ALD and NAFLD patients. In NAFLD, SNPs related to lipid metabolism might play a more important role, whereas SNPs related to alcohol metabolism might be more relevant in ALD. Reduced alcohol-metabolizing activity of alcohol dehydrogenase (ADH1B genotype GG) and increased activity of the aldehyde dehydrogenase (ALDH2 genotype GG) were more frequently observed in ALD patients [20]. Microsomal triglyceride transfer protein (MTP) regulates hepatic triglycerides and very low-density lipoproteins (VLDL) by exporting lipids from the liver. It has recently been shown that the MTP polymorphism genotype GG was associated with NAFLD [44].

\section{The Role of Alcohol in the Development of Obesity and MetS}

Alcohol has a caloric content of $7.1 \mathrm{kcal} / \mathrm{g}$, and calories resulting from alcohol consumption are additive to calories from other sources [45]. However, the evidence linking alcohol consumption with weight gain is not consistent [46]. There are two main types of studies to examine the consumption of alcohol and its effect on weight: a cross-sectional and a longitudinal studies. The general consensus from cross-sectional studies is that in men the alcohol intake is not linked to BMI and in women it is either not linked or there is a negative association [47]. Heavy drinking and binge drinking have been shown to have an association with weight increase and also overweight and an increase in body fat percentage in adolescents or older adults (reviewed in [46]).

Longitudinal prospective studies have been published with follow-up periods of up to 20 years. Heavy drinkers may be more at risk for obesity than light-to-moderate drinkers [48]. In addition, male light-to-moderate drinkers had a lower BMI and waist circumference as compared to nondrinkers or heavy drinkers after 8.5 years of follow-up [49]. In women, however, there was a doseresponse relationship between beer intake and weight gain and waist circumference.

The inconsistency in these studies may be due to confounding features, such as the type of beverage consumed (beer is rich in carbohydrates, for example), different levels of physical activity, and shorter sleep duration (reviewed in [46]).

There is a high prevalence of MetS in AUD. A metaanalysis based on five studies, including 865 individuals, found that more than 1 of 5 patients with AUD had MetS [50]. There was, however, insufficient data to compare 
with populations without AUD. A later analysis of the cross-sectional Indian study of Mattoo et al. [51] observed a similar risk to that of the general population [52]. In contrast, a study from Germany found that the prevalence of MetS in an alcohol-dependent group was nearly twice that of an age-matched, but not gender-matched, control group (31 vs. 17\%) [53]. One reason for this disparity is the presence of psychiatric comorbidity in patients with AUD. Individuals with major depressive disorders, bipolar disease, and post-traumatic stress disease, which are all prevalent in AUD, have an increased risk for MetS [54-56].

In the elderly, both the prevalence and incidence of MetS have been studied in the Italian Longitudinal Study on aging (ILSA) cohort. This included 1,321 men and 1,122 women grouped into five classes of alcohol consumption. The median follow-up was 3.5 years. There was no increase in the prevalence of MetS when compared to abstainers from alcohol for both genders. In the incidence cohort, systolic blood pressure, glycemia, and waist circumference were shown to be affected in men but not in women [57].

\section{The Effect of Alcohol Consumption on Patients with NAFLD}

It is clear that heavy alcohol consumption is detrimental to the liver, including those affected by NAFLD [58]. There is evidence that moderate alcohol consumption (not more than $30 \mathrm{~g} /$ day) reduces the risk of diabetes, hypertension, cardiovascular disease, systemic inflammation, and all-cause mortality rates [59-63]. It has been suggested that there is a J-type curve for moderate alcohol consumption and liver disease, obesity, and cardiovascular health [64]. The authors of this last paper have presented a detailed discussion of the safety of moderate alcohol consumption in overweight individuals [64].

A review on the evidence of the effect of alcohol on NAFLD and histologically diagnosed NAFLD, together with laboratory data on a high-fat diet-induced NASH model in Sprague-Dawley rats, concluded that for those with underlying simple steatosis or $\mathrm{NASH}$, the current data do not support the use of alcohol even at light or moderate amounts [65]. A detailed discussion of this subject is beyond the scope of this review, but we view it as prudent to advise patients with a more advanced disease to abstain from alcohol consumption.

\section{The Impact of Obesity and MetS on ALD}

The prevalence of both obesity and MetS is increasing in the general population. This trend is likewise observed in ALD patients: in the Third National Health and Nutri- tion Examination Survey (NHANCE III) cohort, the prevalence of obesity and components of the MetS was 45 and $32 \%$, respectively [66]. This high prevalence was maintained in a more recent cohort of subjects with ALD, where more than two thirds had central obesity and more than one third had MetS [67].

Obesity is a risk factor for the progression of ALD. Being overweight for at least 10 years is independently correlated with the presence of steatosis, acute alcoholic hepatitis, and cirrhosis [68]. Overweight or obese women in the UK who consume low-to-moderate amounts of alcohol had a higher risk of liver cirrhosis compared to women with a BMI between 22.5 and 25. Data from two prospective cohorts reveal that obesity and alcohol consumption are strongly associated with liver-related mortality. Drinkers of 15 or more units per week have adjusted relative rates for liver disease mortality of 3.16 (95\% confidence interval [CI] 1.28-7.8) for underweight/normal weight men, 7.01 (95\% CI 3.02-16.3) for overweight, and 18.9 (95\% CI 6.84-52.4) for obese men, suggesting that obesity and alcohol consumption synergistically increase mortality in this population [68]. Therefore, the observation that obesity and alcohol interact in promoting liver injury has some pathophysiological support. Obesity and alcohol both reduce adipokine production in visceral adipose tissue, and adiponectin (an anti-fibrotic) is reduced in individuals with obesity or sustained high alcohol consumption $[69,70]$.

In this regard, adiponectin and TNF- $\alpha$ were found to be significantly lower in NAFLD than in cirrhotic or noncirrhotic individuals with ALD [71].

In many subjects, obesity is accompanied by other component of MetS, e.g., dyslipidemia, hypertension, and diabetes. This makes it difficult to conclude whether it is obesity itself or the accompanying metabolic conditions that drive the risk of more advanced liver disease. In a large Finnish study, BMI and waist circumference did not predict severe liver disease [72]. In contrast, a high waist circumference-to-BMI ratio (a high-risk metabolic condition) was a strong driver of the risk for liver events [72]. Among alcohol risk users, the only metabolic factor to predict the risk for liver events was diabetes [72]. Raynard et al. [73] likewise found blood glucose to be an independent risk factor for liver fibrosis in ALD.

A large US study based on the NHANES III survey [66] found that among 235 persons with presumed ALD, independent predictors of liver-related mortality were age, male sex, obesity (elevated BMI and/or waist circumference), and components of MetS. The independent effect of diabetes was almost significant. Interestingly, there was no increased overall mortality risk for either obesity or MetS in this cohort.

Recently, our group has analyzed a cohort of $280 \mathrm{pa}-$ tients with well characterized ALD. A third of this co- 
Fig. 1. The interaction between NAFLD and ALD results in progression to cirrhosis.

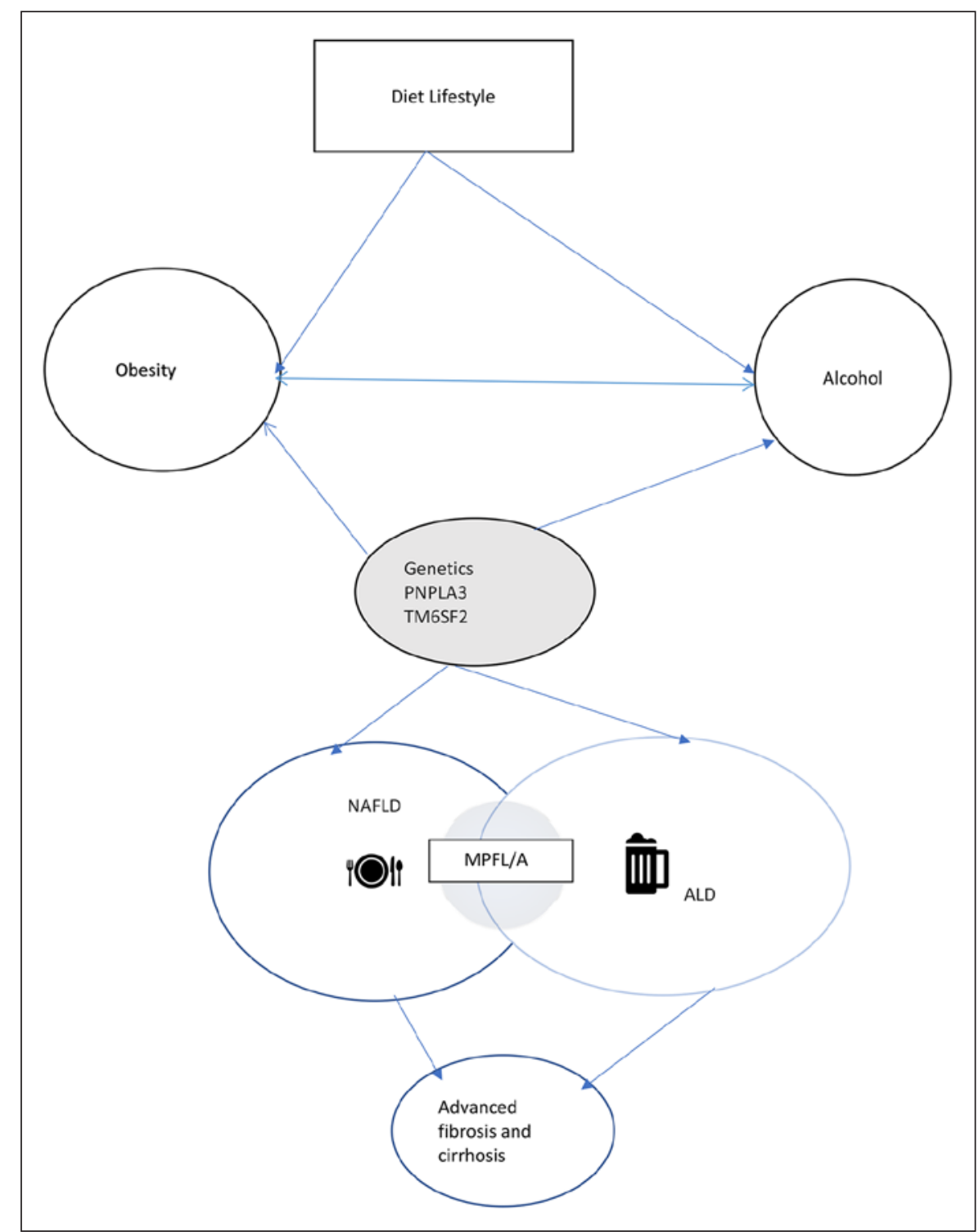

hort was affected with at least two components of MetS, and about $10 \%$ had diabetes. Patients with MetS were older and consumed less alcohol compared to those without MetS. No significant differences were found in the rate of cirrhosis, decompensated liver disease, and HCC between the subgroups; however, patients with ALD and MetS were at an increased risk of cardiovascular disease and nonhepatic malignancy (unpublished data).

Finally, given the enhanced liver injury and fibrosis from concomitant alcohol consumption and obesity [74], it is conceivable that alcohol and obesity may enhance hepatic carcinogenesis. In a population-based study of Taiwanese residents, there was an association between alcohol use and obesity with the risk of HCC incidence [74]. The interaction between the various causes of fatty liver disease is shown in Figure 1.

\section{Conclusions}

It appears that ALD and NAFLD may coexist to varying degrees in a significant number of patients. Both alcohol use and variables of MetS are continuous and not dichotomic variables. The term dual-etiology fatty liver disease [3] may serve as a more relevant diagnostic entity. Risk assessment should be analyzed as a continuum to obtain a picture of the relative contribution of metabolic factors and alcohol use to liver morbidity and mortality. These are also factors to be considered in future risk scores for quantifying individual risk for liver disease. Risk stratification can eventually guide referral practices in primary care, and enable targeted liver diagnostics and follow-up. Risk stratification is becoming more important, as the population prevalence of metabolic abnormalities and fatty liver are increasing. The coexistence of 
these dichotomous processes in patients with fatty liver disease may also raise the awareness to higher-than-recommended alcohol consumption that falls short of a clear ALD diagnosis. In this regard, it becomes mandatory to reappraise several critical issues, such as the hepatotoxic dose thresholds for alcohol and the guideline recommendations for "safe" alcohol consumption, in the presence of overweight/obesity and associated metabolic disease.

\section{Acknowledgement}

Aside from the authors, there are no other individuals or organizations that have made a contribution.

\section{Disclosure Statement}

There authors have no conflicts of interest to disclose.

\section{Funding Sources}

No sources of funding were received.

\section{Author Contributions}

Both authors contributed equally to the planning, writing, and editing of the manuscript.

\section{References}

1 Nassir F, Rector RS, Hammoud GM, Ibdah JA. Pathogenesis and prevention of hepatic steatosis. Gastroenterol Hepatol (N Y). 2015 Mar;11(3):167-75.

2 Sanyal AJ. Past, present and future perspectives in nonalcoholic fatty liver disease. Springer, US: Nature Reviews Gastroenterology and Hepatology; 2019. https://doi. org/10.1038/s41575-019-0144-8.

3 Boyle M, Masson S, Anstee QM. The bidirectional impacts of alcohol consumption and the metabolic syndrome: cofactors for progressive fatty liver disease. J Hepatol. 2018 Feb;68(2):251-67.

4 Rehm J, Mathers C, Popova S, Thavorncharoensap $M$, Teerawattananon Y, Patra J. Global burden of disease and injury and economic cost attributable to alcohol use and alcohol-use disorders. Lancet. 2009 Jun;373(9682):2223-33.

5 Rehm J, Samokhvalov AV, Shield KD. Global burden of alcoholic liver diseases. J Hepat. 2013 Jul;59(1):160-8.

6 Asrani SK, Devarbhavi H, Eaton J, Kamath PS. Burden of liver diseases in the world. J Hepatol. 2019 Jan;70(1):151-71.

7 Bentham J, et al.; NCD Risk Factor Collaboration (NCD-RisC). Worldwide trends in bodymass index, underweight, overweight, and obesity from 1975 to 2016: a pooled analysis of 2416 population-based measurement studies in 128.9 million children, adolescents, and adults. Lancet. 2017 Dec;390(10113):2627-42.

8 Younossi ZM, et al. Changes in the prevalence of the most common causes of chronic liver diseases in the United States from 1988 to 2008. Clin Gastroenterol Hepatol 9, no. 6, pp. 524-530.e1; quiz e60, 2011. https://doi. org/10.4016/scivee.

9 Younossi Z, Anstee QM, Marietti M, Hardy T, Henry L, Eslam M, et al. Global burden of NAFLD and NASH: trends, predictions, risk factors and prevention. Nat Rev Gastroenterol Hepatol. 2018 Jan;15(1):11-20.

10 Rinella M, Charlton M. The globalization of nonalcoholic fatty liver disease: prevalence and impact on world health. Hepatology. 2016 Jul;64(1):19-22.

11 Bugianesi E, McCullough AJ, Marchesini G. Insulin resistance: a metabolic pathway to chronic liver disease. Hepatology. 2005 Nov; 42(5):987-1000.

12 Lonardo A, Nascimbeni F, Targher G, Bernardi M, Bonino F, Bugianesi E, et al.; Italian Association for the Study of the Liver (AISF). AISF position paper on nonalcoholic fatty liver disease (NAFLD): updates and future directions. Dig Liver Dis. 2017 May;49(5):471-83.

13 Younossi ZM, Koenig AB, Abdelatif D, Fazel Y, Henry L, Wymer M. Global epidemiology of nonalcoholic fatty liver disease - Meta-analytic assessment of prevalence, incidence, and outcomes. Hepatology. 2016 Jul;64(1): 73-84.

14 Abdul-Hai A, Abdallah A, Malnick SD. Influence of gut bacteria on development and progression of non-alcoholic fatty liver disease. World J Hepatol. 2015 Jun;7(12):1679-84.

15 Marchesini G, et al.; European Association for the Study of Obesity (EASO). EASL-EASDEASO Clinical Practice Guidelines for the management of non-alcoholic fatty liver disease. J Hepatol. 2016 Jun;64(6):1388-402.

16 Tripathi A, et al. Publisher Correction: The gut-liver axis and the intersection with the microbiome. Nat Rev Gastroenterol Hepatol. 2018 Dec;15(12):785.

17 Mitchell MC, Friedman LS, McClain CJ. Medical Management of Severe Alcoholic Hepatitis: Expert Review from the Clinical Practice Updates Committee of the AGA Institute. Clin Gastroenterol Hepatol. 2017 Jan; 15(1):5-12.

18 Ertle J, Dechêne A, Sowa JP, Penndorf V, Herzer K, Kaiser G, et al. Non-alcoholic fatty liver disease progresses to hepatocellular carcinoma in the absence of apparent cirrhosis. Int J Cancer. 2011 May;128(10):2436-43.

19 Toshikuni N, Tsutsumi M, Arisawa T. Clinical differences between alcoholic liver disease and nonalcoholic fatty liver disease. World J Gastroenterol. 2014 Jul;20(26):8393-406.

20 Celli R, Zhang X. Pathology of Alcoholic Liver Disease. J Clin Transl Hepatol. 2014 Jun; 2(2):103-9.

21 Anstee QM, Seth D, Day CP. Genetic Factors That Affect Risk of Alcoholic and Nonalcoholic Fatty Liver Disease. Gastroenterology. 2016 Jun;150(8):1728-1744.e7.
22 Tripathi A, Debelius J, Brenner DA, Karin M, Loomba R, Schnabl B, et al. The gut-liver axis and the intersection with the microbiome. Nat Rev Gastroenterol Hepatol. 2018 Jul; 15(7):397-411.

23 Bajaj JS. Alcohol, liver disease and the gut microbiota. Nat Rev Gastroenterol Hepatol. 2019 Apr;16(4):235-46.

24 Gillevet P, Sikaroodi M, Keshavarzian A, Mutlu EA. Quantitative assessment of the human gut microbiome using multitag pyrosequencing. Chem Biodivers. 2010 May;7(5): $1065-75$.

25 Rodríguez JM, Murphy K, Stanton C, Ross RP, Kober OI, Juge N, et al. The composition of the gut microbiota throughout life, with an emphasis on early life. Microb Ecol Health Dis. 2015 Feb;26:26050.

26 Drago L, Toscano M, Rodighiero V, De Vecchi E, Mogna G. Cultivable and pyrosequenced fecal microflora in centenarians and young subjects. J Clin Gastroenterol. 2012 Oct;46 Suppl, S81-4.

27 Grant BF, Dufour MC, Harford TC. Epidemiology of alcoholic liver disease. Semin Liver Dis. 1988 Feb;8(1):12-25.

28 de Timary P, Leclercq S, Stärkel P, Delzenne N. A dysbiotic subpopulation of alcohol-dependent subjects. Gut Microbes. 2015;6(6): 388-91.

29 Mutlu EA, Gillevet PM, Rangwala H, Sikaroodi $\mathrm{M}$, Naqvi A, Engen PA, et al. Colonic microbiome is altered in alcoholism. Am J Physiol Gastrointest Liver Physiol. 2012 May; 302(9):G966-78.

30 Leclercq S, Matamoros S, Cani PD, Neyrinck AM, Jamar F, Stärkel P, et al. Intestinal permeability, gut-bacterial dysbiosis, and behavioral markers of alcohol-dependence severity. Proc Natl Acad Sci USA. 2014 Oct; 111(42):E4485-93.

31 Couch RD, Dailey A, Zaidi F, Navarro K, Forsyth CB, Mutlu E, et al. Alcohol induced alterations to the human fecal VOC metabolome. PLoS One. 2015 Mar;10(3):e0119362.

32 Hooper LV, Littman DR, Macpherson AJ. Interactions between the microbiota and the immune system. Science. 2012 Jun;336(6086): 1268-73. 
33 Leclercq S, Stärkel P, Delzenne NM, de Timary $\mathrm{P}$. The gut microbiota: A new target in the management of alcohol dependence? Alcohol. 2019 Feb;74:105-11.

34 Wong VW, Tse CH, Lam TT, Wong GL, Chim AM, Chu WC, et al. Molecular characterization of the fecal microbiota in patients with nonalcoholic steatohepatitis - A longitudinal study. PLoS One. 2013 Apr;8(4):e62885.

35 Le Roy T, Llopis M, Lepage P, Bruneau A, Rabot S, Bevilacqua $\mathrm{C}$, et al. Intestinal microbiota determines development of non-alcoholic fatty liver disease in mice. Gut. 2013 Dec;62(12):1787-94.

36 Boursier J, Mueller O, Barret M, Machado M, Fizanne L, Araujo-Perez F, et al. The severity of nonalcoholic fatty liver disease is associated with gut dysbiosis and shift in the metabolic function of the gut microbiota. Hepatology. 2016 Mar;63(3):764-75.

37 Loomba R, Seguritan V, Li W, Long T, Klitgord N, Bhatt A, et al. Gut Microbiome-Based Metagenomic Signature for Non-invasive Detection of Advanced Fibrosis in Human Nonalcoholic Fatty Liver Disease. Cell Metab. 2017 May;25(5):1054-1062.e5.

38 Rahman K, Desai C, Iyer SS, Thorn NE, Kumar P, Liu Y, et al. Loss of Junctional Adhesion Molecule A Promotes Severe Steatohepatitis in Mice on a Diet High in Saturated Fat, Fructose, and Cholesterol. Gastroenterology. 2016 Oct;151(4):733-746.e12.

39 Sellmann C, Priebs J, Landmann M, Degen C, Engstler AJ, Jin CJ, et al. Diets rich in fructose, fat or fructose and fat alter intestinal barrier function and lead to the development of nonalcoholic fatty liver disease over time. J Nutr Biochem. 2015 Nov;26(11):1183-92.

40 Jiang W, Wu N, Wang X, Chi Y, Zhang Y, Qiu $\mathrm{X}$, et al. Dysbiosis gut microbiota associated with inflammation and impaired mucosal immune function in intestine of humans with non-alcoholic fatty liver disease. Sci Rep. 2015 Feb;5(1):8096.

41 Meroni M, Longo M, Rametta R, Dongiovanni P. Genetic and epigenetic modifiers of alcoholic liver disease. Int J Mol Sci. 2018 Dec; 19(12):E3857.

42 Buch S, Stickel F, Trépo E, Way M, Herrmann A, Nischalke HD, et al. A genome-wide association study confirms PNPLA3 and identifies TM6SF2 and MBOAT7 as risk loci for alcohol-related cirrhosis. Nat Genet. 2015 Dec;47(12):1443-8.

43 Valenti L, Al-Serri A, Daly AK, Galmozzi E Rametta R, Dongiovanni P, et al. Homozygosity for the patatin-like phospholipase-3/adiponutrin I148M polymorphism influences liver fibrosis in patients with nonalcoholic fatty liver disease. Hepatology. 2010 Apr; 51(4):1209-17.

44 Yamamoto K, Kogiso T, Taniai M, Hashimoto E, Tokushige K. Differences in the genetic backgrounds of patients with alcoholic liver disease and non-alcoholic fatty liver disease. JGH Open. 2018 Oct;3(1):17-24.

45 Yeomans MR. Alcohol, appetite and energy balance: is alcohol intake a risk factor for obesity? Physiol Behav. 2010 Apr;100(1):82-9.

46 Traversy G, Chaput JP. Alcohol Consumption and Obesity: an Update. Curr Obes Rep. 2015 Mar;4(1):122-30.
47 Sayon-Orea C, Martinez-Gonzalez MA, BesRastrollo M. Alcohol consumption and body weight: a systematic review. Nutr Rev. 2011 Aug;69(8):419-31.

48 MacInnis RJ, Hodge AM, Dixon HG, Peeters A, Johnson LE, English DR, et al. Predictors of increased body weight and waist circumference for middle-aged adults. Public Health Nutr. 2014 May;17(5):1087-97.

49 Schütze M, Schulz M, Steffen A, Bergmann MM, Kroke A, Lissner L, et al. Beer consumption and the 'beer belly': scientific basis or common belief? Eur J Clin Nutr. 2009 Sep; 63(9):1143-9.

50 Vancampfort D, Hallgren M, Mugisha J, De Hert M, Probst M, Monsieur D, et al. The prevalence of metabolic syndrome in alcohol use disorders: A systematic review and metaanalysis. Alcohol Alcohol. 2016 Sep;51(5): 515-21.

51 Mattoo SK, Nebhinani N, Aggarwal M, Basu D, Kulhara P. Metabolic syndrome among substance dependent men: A study from north India. Ind Psychiatry J. 2013 Jan;22(1): 60-4.

52 Aneja J, Basu D, Mattoo SK, Kohli KK. Metabolic syndrome in alcohol-dependent men: a cross-sectional study. Indian J Psychol Med. 2013 Apr;35(2):190-6.

53 Kahl KG, Greggersen W, Schweiger U, Cordes J, Correll CU, Ristow J, et al. Prevalence of the metabolic syndrome in men and women with alcohol dependence: results from a cross-sectional study during behavioural treatment in a controlled environment. Addiction. 2010 Nov;105(11):1921-7.

54 Vancampfort D, Stubbs B, Mitchell AJ, De Hert M, Wampers M, Ward PB, et al. Risk of metabolic syndrome and its components in people with schizophrenia and related psychotic disorders, bipolar disorder and major depressive disorder: a systematic review and meta-analysis. World Psychiatry. 2015 Oct; 14(3):339-47.

55 Vancampfort D, Vansteelandt K, Correll CU, Mitchell AJ, De Herdt A, Sienaert P, et al. Metabolic syndrome and metabolic abnormalities in bipolar disorder: a meta-analysis of prevalence rates and moderators. Am J Psychiatry. 2013 Mar;170(3):265-74.

56 Rosenbaum S, Stubbs B, Ward PB, Steel Z, Lederman O, Vancampfort D. The prevalence and risk of metabolic syndrome and its components among people with posttraumatic stress disorder: a systematic review and metaanalysis. Metabolism. 2015 Aug;64(8):926-33.

57 Buja A, Scafato E, Sergi G, Maggi S, Suhad MA, Rausa G, et al.; ILSA Working Group. Alcohol consumption and metabolic syndrome in the elderly: results from the Italian longitudinal study on aging. Eur J Clin Nutr. 2010 Mar;64(3):297-307.

58 Rocco A, Compare D, Angrisani D, Sanduzzi Zamparelli M, Nardone G. Alcoholic disease: liver and beyond. World J Gastroenterol. 2014 Oct;20(40):14652-9.

59 Knott C, Bell S, Britton A. Alcohol Consumption and the Risk of Type 2 Diabetes: A Systematic Review and Dose-Response Metaanalysis of More Than 1.9 Million Individuals From 38 Observational Studies. Diabetes Care. 2015 Sep;38(9):1804-12.
60 Briasoulis A, Agarwal V, Messerli FH. Alcohol consumption and the risk of hypertension in men and women: a systematic review and meta-analysis. J Clin Hypertens (Greenwich). 2012 Nov;14(11):792-8.

61 Di Castelnuovo A, Rotondo S, Iacoviello L Donati MB, De Gaetano G. Meta-analysis of wine and beer consumption in relation to vascular risk. Circulation. 2002 Jun; 105(24): 2836-44.

62 Wannamethee SG, Lowe GD, Shaper G, Whincup PH, Rumley A, Walker M, et al. The effects of different alcoholic drinks on lipids, insulin and haemostatic and inflammatory markers in older men. Thromb Haemost. 2003 Dec;90(6):1080-7.

63 Grønbaek M, Johansen D, Becker U, Hein HO, Schnohr P, Jensen G, et al. Changes in alcohol intake and mortality: a longitudinal population-based study. Epidemiology. 2004 Mar;15(2):222-8.

64 Sookoian S, Pirola CJ. How Safe Is Moderate Alcohol Consumption in Overweight and Obese Patients. Gastroenterology. 2016 Jun; 150(8):1698-1703.e2.

65 Seitz HK, Mueller S, Hellerbrand C, Liangpunsakul S. Effect of chronic alcohol consumption on the development and progression of nonalcoholic fatty liver disease (NAFLD). Hepatobiliary Surg Nutr. 2015 Jun;4(3):147-51.

66 Stepanova M, Rafiq N, Younossi ZM. Components of metabolic syndrome are independent predictors of mortality in patients with chronic liver disease: a population-based study. Gut. 2010 Oct;59(10):1410-5.

67 Singh A, Vigni A, Tabbaa A, Scott A, Mansouri $\mathrm{M}$, et al. Increased prevalence of obesity and metabolic syndrome in patients with alcoholic fatty liver. Am J Gastroenterol. 2018 Oct;113:S465.

68 Naveau S, Giraud V, Borotto E, Aubert A, Capron F, Chaput JC. Excess weight risk factor for alcoholic liver disease. Hepatology. 1997 Jan;25(1):108-11.

69 Finelli C, Tarantino G. What is the role of adiponectin in obesity related non-alcoholic fatty liver disease? World J Gastroenterol. 2013 Feb;19(6):802-12.

70 Rogers CQ, Ajmo JM, You M. Adiponectin and alcoholic fatty liver disease. IUBMB Life. 2008 Dec;60(12):790-7.

71 Sowa JP, Atmaca Ö, Kahraman A, Schlattjan M, Lindner M, Sydor S, et al. Non-invasive separation of alcoholic and non-alcoholic liver disease with predictive modeling. PLoS One. 2014 Jul;9(7):e101444.

72 Åberg F, Helenius-Hietala J, Puukka P, Färkkilä M, Jula A. Interaction between alcohol consumption and metabolic syndrome in predicting severe liver disease in the general population. Hepatology. 2018 Jun;67(6): 2141-9.

73 Raynard B, Balian A, Fallik D, Capron F, Bedossa P, Chaput JC, et al. Risk factors of fibrosis in alcohol-induced liver disease. Hepatology. 2002 Mar;35(3):635-8.

74 Loomba R, Yang HI, Su J, Brenner D, BarrettConnor E, Iloeje U, et al. Synergism between obesity and alcohol in increasing the risk of hepatocellular carcinoma: a prospective cohort study. Am J Epidemiol. 2013 Feb;177(4): 333-42.
Interplay between Alcoholic Liver

Disease, Obesity, and MetS 